\title{
Labyrinthe
}

$20 \mid 2005(1)$

La Cognition

\section{Urbanisme et cognition}

Deux tentatives américaines dans les années 1950 et 1960

\section{Clément Orillard}

\section{(2) OpenEdition}

\section{Journals}

Édition électronique

URL : http://journals.openedition.org/labyrinthe/760

DOI : $10.4000 /$ labyrinthe.760

ISSN : 1950-6031

Éditeur

Hermann

\section{Édition imprimée}

Date de publication : 20 avril 2005

Pagination : 77-32

\section{Référence électronique}

Clément Orillard, «Urbanisme et cognition », Labyrinthe [En ligne], 20 | 2005 (1), mis en ligne le 26 juin 2008, consulté le 01 mai 2019. URL : http://journals.openedition.org/labyrinthe/760 ; DOI : 10.4000/ labyrinthe.760

Propriété intellectuelle 


\title{
URBANISME ET COGNITION \\ Deux tentatives américaines dans les années 1950 et 1960
}

\author{
Clément ORILLARD \\ orillard@aol.com
}

L'urbanisme n'est pas un champ du savoir qui pourrait prétendre à une quelconque unité et donc au statut de discipline, mais au contraire un vaste ensemble hétérogène de pratiques, de savoir-faire et de savoirs qui portent sur le même objet: la ville. Parler d'approche cognitiviste en ce qui concerne l'urbanisme revient donc à rechercher parmi les divers écrits théoriques portant sur la ville, ceux qui font explicitement référence à certains traits caractéristiques de l'approche cognitiviste des sciences sociales.

Il est difficile d'isoler dans l'histoire de l'urbanisme ce que l'on pourrait appeler un courant cognitif. Néanmoins, au sein de l'océan des écrits qui s'inscrivent dans ce champ, émergent deux auteurs qui ont tenté de formuler ce qui pourrait être qualifié d' «urbanisme cognitif». Kevin Lynch et Christopher Alexander, architectes de formation mais quelque peu hétérodoxes, furent enseignants toute leur vie. Pourtant, malgré cette approche cognitive des projets urbains, leurs œuvres diffèrent nettement. Nous allons voir que, sans former un courant particulier de l'histoire de l'urbanisme, ces deux auteurs s'inscrivent dans un cadre commun qui les rapproche d'une manière étonnante. Mais que les modalités de cette inscription expliquent aussi les différences que tout lecteur constate dans leurs écrits.

\section{Dynamiques professionnelles au sein de l'urbanisme américain : concurrence et rationalisation}

Avant d'aborder le contexte plus particulier qui a présidé au travail de Kevin Lynch et de Christopher Alexander, il est nécessaire de comprendre les dynamiques plus vastes touchant le monde de l'urbanisme améri- 


\section{Labyrinthe, $n^{\circ} 20$}

cain durant la première moitié du siècle dernier. Ces dynamiques sont essentiellement professionnelles et liées à un mouvement généralisé de rationalisation et de scientifisation de la gestion des problèmes urbains.

\section{Des architectes aux planners : rationalisation de la réforme urbaine}

La Première Guerre mondiale provoque aux USA, une rupture dans le champ urbanistique tant au niveau des professions qu'à celui des pratiques. Jusqu'à cette date, le modèle urbanistique dominant aux États-Unis est celui du mouvement du City Beautiful dont la figure centrale est Daniel Burnham. Ce modèle forgé à l'occasion de l'exposition universelle de Chicago est réformiste, porté par une profession alors en pleine structuration, les architectes; il est fondé sur un vocabulaire formel hérité de la grande tradition de l'embellissement urbain qui s'oppose violemment aux formes de la grande croissance urbaine de la fin du XIX siècle. Ce modèle est en fait la traduction locale du modèle qui domine la réflexion urbanistique au tournant du siècle: le modèle Beaux-Arts promu par l'école du même nom. Cette traduction s'est développée à travers l'existence au sein de l'École nationale supérieure des beaux-arts de l' «atelier américain» qui assure un complément de formation à des étudiants déjà formés dans des agences américaines. Le passage par cet atelier est, jusqu'en 1917, indispensable pour obtenir la renommée qui doit permettre d'accéder à la grande commande ${ }^{1}$. Pourtant, dès la fin du XIX ${ }^{e}$ siècle se met en place un enseignement universitaire en architecture; mais là encore le modèle reste celui de l'atelier Beaux-Arts. Les quatre ans de la Première Guerre mondiale mettent sérieusement à mal ce modèle. D'un côté, la guerre a interrompu la circulation entre les États-Unis et la France des étudiants. D'un autre, l'image de la France a profondément souffert de la guerre alors que les États-Unis en sortent renforcés.

C'est dans ce contexte que le modèle urbanistique dominant aux USA va changer. D'un modèle exogène et lié à la profession d'architecte, on passe à un modèle endogène et lié à d'autres professions. Ce

1. Voir Joan Draper, «The École des beaux-arts and the Architectural Profession in the United States : the Case of John Galen Howard» dans Spiro Kostof (dir.), The Architect. Chapters in History of the Profession, New York, Oxford University Press, 1977. 


\section{Urbanisme et cognition}

nouveau modèle est lié au mouvement de réforme urbaine qui naît aux États-Unis au début du siècle. Il se présente d'abord comme un mouvement civique de réforme sociale comme le City Beautiful, mais surtout de réforme esthétique de la ville. D'abord hétérogène, il se structure dès 1909 avec la naissance de la National Conference on City Planning, puis en 1917 avec l'American City Planning Institute. Durant la même période des cursus de City Planning naissent au sein des principales universités américaines. C'est ainsi que les questions urbaines deviennent le domaine dès les années 1920 d'une profession nouvelle: les planners. À l'origine, les liens restent forts entre le City Planning et la profession d'architecte: l'ACPI est fondé sur le modèle de l'American Institute of Architects, les formations sont souvent développées au sein des Schools of Architecture des différentes universités ${ }^{2}$. Mais rapidement, cette proximité s'estompe. Ainsi les troupes qui viennent rejoindre cette profession ne sont pas majoritairement issues des rangs de l'architecture. Un corps occupe une place très importante, celui des architectes-paysagistes (landscape architects). Alors que ces derniers dominent de plus en plus le marché de l'urbanisme en proposant des outils à même de répondre à une demande civique se portant sur des échelles toujours plus grandes, ils restent membres d'une profession peu organisée. Ils se glisseront ainsi très facilement dans ce nouveau costume taillé à leur mesure, celui du planner, consultant pour des groupes civiques locaux ${ }^{3}$. Ils sont, de plus, porteurs d'un vocabulaire urbanistique nouveau articulé autour du trio cité jardins-parkwaysparcs qui deviendra la doxa des plans d'aménagement d'avant-guerre. Mais la Seconde Guerre mondiale voit de nouveau une rupture avec l'institutionnalisation de la profession de planners. D'un côté, cette dernière perd peu à peu son héritage paysager pour intégrer un discours de plus en plus techno-scientifique et quantitatif ${ }^{4}$. D'un autre, elle

\footnotetext{
2. Sur la naissance des institutions liées au planning, voir Eugenie Ladner Birch, «Advancing the Art and Science of Planning. Planners and Their Organizations. 1909-1980», Journal of the American Planning Association, vol. 46, $\mathrm{n}^{\circ} 1$, janvier 1980, p. 22-49.

3. Voir Christian Topalov, «L'Urbanisme comme mouvement social. Militants et professionnels du City Planning aux États-Unis (1909-1917)», Annales de la recherche urbaine, ${ }^{\circ}$ 44-45, décembre 1989, p. 139-154.

4. Sur cette «technicisation » du discours des planners, voir Cliff Elis, «Professional Conflict over Urban Form. The Case of Urban Freeways, 1930 to 1970 », dans Mary Corbin Sies \& Christopher Silver (dir.), Planning the Twentieth-Century American City, Baltimore, The Johns Hopkins University Press, 1996, p. 262-279.
} 
intègre massivement les nouveaux organismes municipaux au service d'édiles bien décidés à transformer les centres-villes. On voit donc que la profession des planners acquiert dans l'immédiat après-guerre une hégémonie définitive ainsi qu'une culture qui éloigne les architectes de la grande commande urbaine, celle du plan de villes.

\section{Vers une scientifisation du discours esthétique des architectes}

Mais la profession des planners n'est pas la seule à connaître la mise en place d'une rationalisation et d'une scientifisation de son discours. Dès l'entre-deux-guerres, alors que le modèle Beaux-Arts commence à reculer nettement dans la plupart des universités américaines, une approche moins stylistique et plus rationnelle du projet architectural se met en place ${ }^{6}$. Cette rationalisation qui rejette le modèle Beaux-Arts est bien sûr en grande partie due à l'émigration aux ÉtatsUnis d'un certain nombre des théoriciens de l' «architecture moderne» et en particulier des enseignants du Bauhaus. Or, il ne faut pas oublier qu'une des ambitions les plus fortes du mouvement moderne en architecture, le cœur de toute une activité réflexive qui permit à ce collectif de se constituer en groupe de pression et de propagande puissant, fut de porter un discours réformiste sur la ville ${ }^{7}$. Cette action restait possible en Europe continentale du fait que le champ de l'urbanisme n'avait pas été accaparé par une profession mais restait un des domaines majeurs d'action théorique mais aussi pratique de nombreux architectes. Et que découvrent ces derniers à leur arrivée sur le territoire américain? Que les questions urbaines échappent aux architectes. Loin d'abandonner leurs ambitions urbanistiques, ils vont, aux côtés d'autres membres autochtones de la profession, chercher à renouveler leur

\footnotetext{
5. Il est à noter - et ceci est très important pour la suite de notre propos - qu'à la différence de la France qui adopte le terme générique d'«urbanisme» pour désigner les pratiques urbanistiques comme les autres pays de l'Europe continentale, les Américains, comme les Britanniques, lui préfèreront le terme « urban planning » désignant explicitement une pratique particulière liée à une profession particulière (la profession des planners est institutionnalisée aussi en Grande-Bretagne). 6. Voir David Brain, « La naissance d'une discipline moderne du projet aux États-Unis. La conception des logements sociaux au début du $\mathrm{xx}^{\mathrm{e}}$ siècle ", Les Cahiers de la recherche architecturale et urbaine, $\mathrm{n}^{\circ} 2-3$, p. $95-106$.

7. Sur le discours des architectes modernes sur la ville et ses évolutions, voir Eric Mumford, The CIAM Discourse on Urbanism, Cambridge (Mass.), The MIT Press, 2002.
} 
approche des questions urbaines, sortir de l'embellissement urbain pour proposer un discours nouveau permettant de répondre aux attentes des édiles, mais aussi à un plus large problème.

On assiste ainsi à partir des années 1950 à un extraordinaire foisonnement théorique du côté des architectes américains. Toutes ces théories tentent de "scientificiser», de rationaliser le discours des architectes sur la forme urbaine dans un registre tout à la fois complémentaire mais aussi concurrent du discours de l'urban planning. À partir des années 1960, ce foisonnement explose et les diverses tentatives pour formuler ce type de discours se voient consacrées par la mise en place de cursus doubles associant enseignement d'urban planning et d'architecture ou de paysagisme et d'architecture, et donc la mise en place d'une pseudo-discipline qui se présente comme complémentaire de l'urban planning et qui prend rapidement le nom d'urban design'. C'est dans ce cadre que se situe l'apparition des deux tentatives de formulation d'un discours urbanistique faisant appel à l'approche cognitiviste.

\section{Le lieu de la rationalisation du discours esthétique: le Massachusetts Institute of Technology}

Il est remarquable que si ces deux tentatives sont décalées dans le temps et ont toutes deux une postérité différente, elles sont liées à un lieu bien particulier qui a joué un rôle majeur dans la scientifisation du discours esthétique évoquée précédemment: le Massachusetts Institute of Technology.

Nombreux sont les cogniticiens mais aussi les historiens des sciences qui s'accordent pour dater la naissance de la «science cognitive $^{9}{ }^{»}$ entre les 10 et 12 septembre 1956, dates du colloque sur les

\footnotetext{
8. Sur les débuts de cette pseudo-discipline, voir David Smiley, «A Tale of Two Conferences: Urban Design and Urban Discourse in the mid-20 $0^{\text {th }}$ Century », dans Urban Design: Practices, Pedagogies, Premises, framing papers for panel discussions held at Columbia University and Lighthouse International, New York, april 5-6, 2002, p. 15-23. Il faut remarquer que c'est une des grandes figures de l'urbanisme moderne européen, Josep Luis Sert, qui assurera au terme «urban design» sa popularité en organisant une conférence sur ce thème à Harvard en 1956.

9. Howard Gardner, historien, mais aussi cogniticien, parle d'un consensus de la communauté en ce qui concerne cette date. Voir Howard Gardner, The Mind's New Science. A History of the Cognitive Revolution, New York, Basic Books, 1985 (traduction française Histoire de la révolution cognitive. La nouvelle science de l'esprit, Paris, Payot, 1993).
} 
Sciences de l'information organisé au MIT. Mais la présence du MIT aux fonts baptismaux de la cognition est loin d'être le fruit du hasard. Au contraire, l'histoire même de cette institution universitaire la prédestinait à accueillir les premières tentatives pour formuler cette rationalité abstraite des processus étudiés par les sciences sociales.

Dans le système universitaire complexe qui se met en place au $\mathrm{XIX}^{\mathrm{e}}$ siècle aux États-Unis ${ }^{10}$, le MIT se positionne comme un institut de technologie fondé sur le modèle de l'École polytechnique française. À la différence des universités fondées sur le modèle humaniste allemand, il dispense un enseignement essentiellement technique, même s'il possède une faculté d'architecture qui est la plus ancienne du pays et aussi un département de philosophie qui disparaît néanmoins rapidement. En fait, face à la demande alors limitée en matière d'enseignement supérieur, le MIT entend être cette institution complémentaire de la grande université d'Harvard et pourvoyeuse des cadres dont l'industrie bostonienne naissante a besoin.

Ce n'est que tardivement que le MIT va voir son statut changer. Déjà, lors de la Première Guerre mondiale, l'institut se rapproche du gouvernement fédéral et de l'armée afin de répondre aux besoins en innovation technologique. Une ébauche de complexe militaro-scientifique se met en place, qui ne survivra pas à l'isolationnisme de l'entre-deuxguerres. Mais lorsque les États-Unis entrent dans la Seconde Guerre mondiale, le gouvernement fédéral fait de nouveau appel aux services des chercheurs du MIT pour gagner la guerre technologique. Les investissements à la hauteur des nécessités sont cette fois-ci sans commune mesure avec le passé. Alors qu'un vrai complexe militaro-industriel se met en place, le MIT en occupe bien vite le cœur ${ }^{11}$. Au sortir de la guerre, l'institut a gagné une place sans égale au sein du gouvernement fédéral et alors que le système des fondations acquiert sa puissance de croisière, il va connaître une croissance sans précédent. C'est ainsi que le MIT, d'institut technologique devient une université généraliste. En 1947, une

10. Sur l'histoire de la recherche universitaire américaine voir Roger L. Geiger, To Advance Knowledge, The Gowth of American Research University, 1900-1940, New York, Oxford University Press, 1986 et Roger L. Geiger, Research and Relevant Knowledge, American Research Universities Since World War II, New York, Oxford University Press, 1993.

11. Le MIT, à travers le Radiation Laboratory, est notamment un des lieux de «l'invention » du radar. Sur les liens entre le MIT et le complexe militaro-industriel, voir Dorothy Nelkin, The University and Military Research: Moral Politics at MIT, Ithaca (N.Y.), Cornell University Press, 1972. 
faculté de lettres et sciences humaines (School of Humanities) est ouverte en son sein et l'architecte, ingénieur et historien John Ely Burchard en devient le premier doyen. Si cette nouvelle faculté ouvre soudainement le champ de l'enseignement dispensé au MIT, elle reste bien sûr très fortement marquée par la tradition techno-scientifique de ce même MIT, une tendance qui se trouve alors en conjonction certaine avec l'atmosphère d'une époque nettement marquée par la domination de ce même paradigme techno-scientifique ${ }^{12}$.

Le développement du MIT ainsi que l'affirmation de plus en plus forte du paradigme techno-scientifique dans la recherche américaine vont pousser l'université d'Harvard à réagir et à orienter sa politique de recherche dans des voies similaires. Ainsi en 1960, Jerome Brunner et George Miller fondent le Harvard Center for Cognitive Studies. La proximité tout autant géographique que méthodologique des recherches effectuées dans les deux grandes institutions universitaires de Cambridge, Massachusetts, va aboutir à un mariage de raison très vigoureusement encouragé par les fondations. La circulation des doctorants mais aussi des enseignants entre les deux établissements devient courante dès la fin des années 1950 alors que des organismes de recherche communs sont créés.

\section{Kevin Lynch et l'image de la ville}

La première tentative de formuler un urbanisme cognitif fut donc celle de Kevin Lynch. Diplômé en 1947 en City Planning de la School of Architecture and City Planning du MIT, il se présente comme un théoricien de l'urbanisme quelque peu hétérodoxe. Issu de Chicago où il a suivi un enseignement secondaire dans une High School alternative, il décide d'étudier l'architecture pour travailler sur les questions urbaines, et effectue un an de scolarité à la School of Architecture de Yale puis une autre année auprès de l'architecte Frank Lloyd Wright dans son atelier de Taliesin. Mais heurté par l'archaïsme de l'enseignement Beaux-Arts encore dispensé à Yale à la fin des années 1930 puis par l'idéologie anti-urbaine professée par Frank Lloyd Wright, il se tourne

12. Le MIT est aussi un des lieux de naissance d'une forme nouvelle d'acteur, le chercheur-entrepreneur, dont le modèle va essaimer dans l'ensemble du monde de la recherche scientifique américaine. 
vers des études d'ingénierie puis de biologie. La guerre interrompt cette évolution et lorsque, avec sa fin et le GI's Bill, la question des études universitaires resurgit, Lynch revient vers les questions urbaines en entrant au $\mathrm{MIT}^{13}$.

Sa vocation d'enseignant est le fruit du hasard mais un fruit qui se révèlera très généreux. Entré comme instructor en 1948 au sein de la School of Architecture and City Planning du MIT, il grimpe rapidement les échelons. En 1951 avec Gyorgy Kepes, il lance un séminaire expérimental nommé «Form of the City » dont le but explicite est de reformuler la question de l'esthétique urbaine. En 1954, l'administration de la faculté d'architecture et d'urbanisme cherche à mettre en place un programme de recherche permettant à cette faculté de conquérir une place centrale au sein des autres universités en matière de questions urbaines. Elle décide de se baser sur les conclusions du séminaire de 1951. C'est ainsi que, entre 1954 et 1959, Kepes et Lynch, puis Lynch seul, se retrouvent à la tête d'un programme très important financé par la fondation Rockefeller qui explore différentes voies dans la construction d'un discours scientifique sur la forme urbaine. Et parmi ces voies, triomphera l'approche cognitive à travers le seul ouvrage issu de cette recherche, The Image of the City ${ }^{14}$.

L'importance de l'approche cognitive est issue de l'enseignement de Gyorgy Kepes. Avant d'être professeur de visual studies à la School of Architecture du MIT depuis 1944, il fut le bras droit de Laszlo Moholy-Nagy, un des principaux théoriciens et enseignants du Bauhaus jusqu'à sa fermeture. $\mathrm{Si}$, à cette époque, il n'enseigne pas mais participe à l'activité privée de Moholy-Nagy, il devient un acteur de premier plan lors de la fondation du New Bauhaus à Chicago en 1937 où il développe un enseignement fondé sur la vision. Une expérience qui aboutit en 1944 à la publication de Language of Vision ${ }^{15}$ puis l'année suivante à son entrée au MIT.

Héritier du Bauhaus, Gyorgy Kepes appréhende donc la question de l'esthétique essentiellement à travers la vision dans une approche

\footnotetext{
13. Sur la biographie de Kevin Lynch voir Tribid Banerjee \& Michael Southworth, « Kevin Lynch: His Life and Work » dans Tribid Banerjee \& Michael Southworth (dir.), City Sense and City Design. Writings and Projects of Kevin Lynch, Cambridge (Mass.), The MIT Press, 1990, p. 1-29.

14. Kevin Lynch, Cambridge (Mass.), The MIT Press, 1960 (traduction française : L'Image de la cité, Paris, Dunod, 1971).

15. De Gyorgy Kepes, New York, Paul Theobald, 1944.
} 


\section{Urbanisme et cognition}

fortement teintée de gestaltisme. Pour lui, parler de forme est avant tout parler de perception. Mais il dépasse cette approche en formulant un projet très ambitieux qui apparaît en 1951 lors de l'exposition The New Landscape in Art and Science ${ }^{16}$. Il pense également que le savoir scientifique a considérablement développé les capacités humaines alors que l'expérience esthétique reste enfermée dans des schémas anciens. Il est donc nécessaire de formuler de nouveaux schémas, et le séminaire expérimental qu'il dirige avec Kevin Lynch la même année participe de cet objectif.

La recherche de nouveaux modes d'approche de la question de la forme urbaine dans le programme de recherche financé par la fondation Rockefeller va suivre diverses pistes. Mais, assez rapidement, Lynch qui reste seul aux commandes concentre le travail sur la question de la représentation mentale. Lors de son voyage d'étude en Europe en 1953-1954, il avait développé deux démarches: une approche quasi-ethnographique nourrie par des carnets d'observation ainsi qu'une approche plus classique avec la réalisation de quelques cartes très simples tentant de saisir de manière très empirique les éléments les plus évidents de la topographie d'une ville. Le programme de recherche va lui permettre de faire converger son intérêt pour l'apport des sciences sociales vers son attachement aux moyens plus classiques de représentation. Dès les premiers travaux, l'approche de la forme urbaine développée par Lynch se teinte fortement de psychologie. S'il continue à travailler à partir de la production d'une cartographie, cette dernière n'a plus pour but de saisir les principaux éléments objectifs de la ville considérée mais de saisir l'image mentale qu'elle suscite chez ses habitants. À partir de 1955, Lynch, en association avec David Crane, alors professeur à l'université de Pennsylvanie, recherche le vocabulaire formel qui doit permettre de décrire au mieux cette image mentale ainsi que la méthode la plus efficace pour la saisir.

Cinq ans plus tard, l'ouvrage publié par le Joint Center for Urban Studies, The Image of the City, fixe la réflexion menée par Kevin Lynch. L'image de la ville est caractérisée à travers trois composantes : identité, structure et signification. Le postulat développé dans l'ouvrage est simple: plus l'image de cette ville est forte et claire, plus la qualité

16. À partir de cette exposition organisée au MIT, il éditera un ouvrage. Voir Gyorgy Kepes, The New Landscape in Art and Science, New York, Paul Theobald, 1956. 
de la forme de cette ville est grande. Afin de décrire cette qualité particulière de la forme urbaine, la notion d'imagibilité est définie:

« C'est, pour un objet physique, la qualité grâce à laquelle il a de grandes chances de provoquer une forte image chez n'importe quel observateur. C'est cette forme, cette couleur ou cette disposition, qui facilitent la création d'images mentales de l'environnement vivement identifiées, puissamment structurée et d'une grande utilité ${ }^{17}$. »

Ensuite, l'auteur développe les éléments composant l'image mentale de la ville. Cinq catégories sont fixées: parcours, limites, nœuds, districts et points de repères. Ces catégories ne sont pas attachées à la nature, ni à l'échelle des objets composant la ville mais sont au contraire construites à partir de la représentation des habitants et usagers de la ville.

Enfin, il indique la méthodologie suivie: l'enquête sur place et les interviews. L'enquête sur place permet de reconnaître les différents éléments objectifs de la forme de la ville qui pourront par la suite correspondre à telle ou telle catégorie. Les interviews viennent fournir les éléments qui permettront de cartographier par la suite l'image mentale de la ville. Ces interviews sont basées sur un questionnaire précis et concis construit à partir de l'enquête sur place. Elles comportent en particulier la production d'une carte schématique par l'interviewé.

L'ouvrage développe la démarche et ses possibles apports à l'aménagement urbain à travers la comparaison de trois villes américaines: Boston, Jersey City et Los Angeles.

\section{Christopher Alexander et la synthèse de la forme}

Christopher Alexander fut le deuxième auteur à développer une approche cognitiviste de l'urbanisme. Si celle de Kevin Lynch reste encore aux marges de l'univers de la cognition, Alexander s'y est résolument immergé.

Christopher Alexander eut un parcours encore plus hétérodoxe que Kevin Lynch, et dès le départ semble particulièrement prédisposé à embrasser les thèses cognitivistes. Né à Vienne, il grandit en Angleterre

17. Kevin Lynch, L'Image de la cité. op. cit., p. 11. 
puis entre à Trinity College à l'université de Cambridge pour étudier la chimie et la physique avant de se tourner vers les mathématiques. Brillant étudiant, c'est toujours à Cambridge qu'il obtient un Master's degree en mathématiques mais aussi un Bachelor's degree en architecture. Dès cette époque, ses recherches s'orientent vers la question de la perception et une approche psychologique de l'esthétique visuelle.

Armé de ce double bagage, il traverse l'Atlantique en 1961 pour poursuivre ses études. Il rejoint Harvard et le MIT. À Harvard où il devient le premier étudiant à poursuivre un doctorat en architecture, il poursuit des recherches en sciences cognitives et rencontre Serge Chermayeff, architecte, artiste et professeur, lui-même immigré ayant par la suite acquis la nationalité britannique. Au MIT, il travaille dans le Civil Engineering System Laboratory en abordant la question des systèmes de transports à partir de l'informatique.

Ses intérêts vont rencontrer ceux de Chermayeff. Ce dernier, en effet, est issu comme Kepes de la première génération des architectes modernes et de leur tentative de rationalisation du discours esthétique. De même connaissait-il Laszlo Moholy-Nagy et est-il passé par le New Bauhaus de Chicago dont il a assumé la direction à la mort de MoholyNagy, avant de rejoindre le MIT puis Harvard à partir de 1952.

C'est donc entre Harvard et le MIT et d'abord sous la direction de Chermayeff qu'Alexander commencera à faire converger sa formation architecturale et mathématicienne à travers des recherches mêlant sciences cognitives et cybernétique. Ses deux premiers ouvrages: Community and Privacy, Toward a New Architecture of Humanism, publié avec Chermayeff, et Notes on the Synthesis of Form ${ }^{18}$ datent de cette période. Alexander y constate les insuffisances des méthodes traditionnelles de conception et la dégradation de l'environnement humain, ce qui rend urgente une refondation de la conception architecturale. La procédure développée consiste en une division du programme auquel le concepteur doit répondre en autant de propositions élémentaires, nommée «ensemble», composant la «structure» de ce problème. La résolution du problème passe ensuite par l'analyse des

\footnotetext{
18. Serge Chermayeff \& Christopher Alexander, Community and Privacy, Toward a New Architecture of Humanism, New York, Doubleday and Company, 1963 (traduction française: Intimité et vie communautaire. Vers un nouvel humanisme architectural, Paris, Dunod, 1972) et Christopher Alexander, Notes on the Synthesis of Form, Cambridge (Mass.), Havard University Press, 1964 (De la synthèse de la forme, Paris, Dunod, 1971).
} 
différentes interactions possibles entre les ensembles. C'est dans cette analyse que l'ordinateur trouve toute sa place: il permet de faire des choix pour trouver les interactions optimales permettant de formuler des composants qui répondent à plusieurs propositions issues du problème. Ces composants sont représentés sous la forme de « diagrammes » (patterns) qui «ne sont pas de simples illustrations ou accompagnement du texte. Ce sont des énoncés schématiques, conquis de haute lutte après un travail méticuleux, qui résument (visuellement) les implications physiques des composants variés des problèmes ${ }^{19}{ }$. Ce faisant, il déconnecte la réponse formelle aux fonctions des fonctions elles-mêmes. Un diagramme correspond à différents sous-ensembles alors qu'un sous-ensemble est lié à diverses fonctions. C'est ainsi qu'Alexander peut affirmer dans un article resté célèbre que «la ville n'est pas un arbre», la ville n'est pas la combinaison ensemble d'éléments strictement fonctionnels.

En fait, cette technique revient à déconstruire la représentation mentale forcément subjective des concepteurs en un ensemble de propositions logiques mais abstraites sur laquelle une science de la conception de la forme peut être basée. Pour formuler ces propositions logiques, Alexander utilise massivement son savoir de mathématicien. Explicitement, son but est de faire rentrer les mathématiques dans le monde de la conception des formes et donc plus précisément de l'architecture.

À Berkeley qu'il rejoint en tant qu'enseignant en 1963, il fonde le Center for Environmental Design. C'est au sein de cette structure qu'il continue de développer une approche cognitiviste de la conception. Il cherche en particulier à développer les diagrammes qui sont à la base de la conception de la forme. Avec son équipe de chercheurs et d'étudiants, un recensement exhaustif de ces patterns est réalisé afin de mettre au point un véritable vocabulaire correspondant aux différents langages possibles de la conception. C'est cette recherche qui aboutit en 1977 à la publication de A Pattern Language ${ }^{20}$ qui range les différents diagrammes possibles sous trois catégories d'échelles: villes, bâtiments, construction. Mais cet ouvrage qui est inclus dans une série

19. Serge Chermayeff \& Christopher Alexander, op. cit., p. 169.

20. Christopher Alexander, Sara Ishikawa \& Murray Silverstein avec Max Jacobson, Ingrid Fiksdahl King \& Shlomo Angel, A Pattern Language. Towns, Buildinds, Construction, New York, Oxford University Press, 1977. 


\section{Urbanisme et cognition}

de trois, sert de base à un autre axe de travail qui s'éloigne de la recherche pure pour rejoindre l'idéologie. En ces années 1970 promptes à donner espoir à toute utopie, les membres du Center for Environmental Design tentent de dissoudre la conception architecturale dans l'autoconstruction ${ }^{21}$.

\section{Comparaison et postérité}

Nous avons vu que les œuvres de Kevin Lynch et Christopher Alexander partagent tout à la fois un contexte général et un lieu de naissance commun. Ce dernier est un lieu central dans le développement de l'approche cognitive. Mais qu'en est-il dans les œuvres elles-mêmes?

Nous retrouvons chez chacun des thèmes liés à l'approche cognitive. Tout d'abord, l'importance donnée à la représentation mentale. Dans le cas du travail de Lynch cette importance est évidente puisqu'elle est au cœur de son discours. Mais Alexander base lui aussi son approche sur l'image mentale. Simplement, il ne s'agit pas de celles des habitants d'une ville mais de celle du concepteur lui-même ${ }^{22}$. Mais au-delà de cette simple affirmation, les deux auteurs pensent cette représentation mentale comme un énoncé pouvant être décomposé à travers un vocabulaire abstrait, symbolique. Nous le voyons chez Lynch avec les cinq éléments correspondant à l'image de la ville mais aussi avec Alexander et ses diagrammes ou patterns.

Or, pour Howard Gardner, la représentation mentale est la première de ce qu'il nomme les «caractéristiques clés de la science cognitive»:

« La science cognitive est basée sur le fait qu'il est légitime, et en fait nécessaire, d'établir un niveau d'analyse que l'on peut appeler "niveau de représentation". Lorsqu'il travaille à ce niveau, un scientifique manipule des entités telles que des symboles, des règles, des images, qui sont la matière de représentation que l'on trouve entre l'entrée (input) et la sortie (output); il explore en outre les manières selon lesquelles ces entités de représentation sont reliées, transformées ou opposées les

\footnotetext{
21. Pour plus de renseignements sur l'ensemble de l'œuvre de Christopher Alexander, voir l'ouvrage plutôt hagiographique que critique de Stephen Grabow, Christopher Alexander: the Search for a New Paradigm in Architecture, Boston, Oriel Press, 1983.

22. Voir Christopher Alexander, op. cit., p. 63-64.
} 
unes aux autres. Ce niveau est nécessaire pour expliquer la diversité du comportement humain, de l'action et de la pensée humaine ${ }^{23}$.»

On retrouve aussi chez Kevin Lynch et Christopher Alexander la même importance réduite accordée aux aspects véhiculés par la tradition sociologique. La représentation mentale est abordée comme un niveau structurel du cerveau humain et non comme une production historique. Elle semble liée à des capacités innées et non à des processus acquis, et paraît transcender les limites culturelles. Nous retrouvons ici la deuxième caractéristique clé de Gardner qui est au fondement du travail de nombreux cogniticiens comme Noam Chomsky. De même, Lynch et Alexander sont-ils attachés à une transdisciplinarité évidente dans leur parcours et dans leurs œuvres mais qui se justifie par le projet de scientifisation de l'architecture.

En revanche, ce qui sépare le travail de Kevin Lynch et celui de Christopher Alexander est l'importance donnée au modèle de l'informatique. Kevin Lynch n'accordera jamais son attention à cet outil dans la résolution des problèmes urbanistiques. Si certains de ses très proches étudiants s'intéresseront à l'ordinateur, son bras droit Donald Appleyard, malgré ses tentatives de plus en plus complexes de construire un langage urbanistique, ne l'utilisera pas, que ce soit au MIT ou à partir de 1967 à Berkeley. Or, comme nous l'avons vu, l'ordinateur occupe une place centrale dans le travail de Christopher Alexander dès le début de ses écrits théoriques, et ces derniers sont impensables sans son usage.

Une des raisons de cette différence est probablement la différence de chronologie entre le travail de Kevin Lynch et celui de Christopher Alexander. Mais ce n'est pas suffisant. Une autre raison, qui semble plus importante, est la différence de parcours entre ces deux auteurs. Si Lynch a commencé un cursus au sein des sciences dures, la guerre a interrompu ce dernier et ces sciences étaient expérimentales - ingénierie et biologie -, alors que, Alexander, après avoir commencé par la physique et la chimie s'est tourné vers les mathématiques dont il a fait la matière principale de son cursus à l'université de Cambridge, l'architecture étant secondaire.

23. Howard Gardner, op. cit., p. 54. 


\section{Urbanisme et cognition}

Maintenant abordons une dernière question: quelle fut la postérité de ces théories? La réponse est paradoxale.

$\mathrm{Au}$ niveau de l'urbanisme, elle fut faible. Les raisons sont nombreuses: structurelles et conjoncturelles. Une des raisons majeures fut probablement leur trop grande rupture avec des pratiques qui caractérisaient la culture architecturale. Remettre en cause ces pratiques d'une manière trop violente n'entraînait-il pas une remise en cause de la profession d'architecte elle-même? La position antiprofessionnelle qu'Alexander a adoptée à Berkeley confirme d'ailleurs ce problème. Une autre raison structurelle est bien sûr les limites de l'approche cognitiviste elle-même. Les postulats innéistes et universalistes proposés restent très contestables. C'est en particulier le cas de l'idée que la représentation mentale serait un objet stable et lié directement à la structure du cerveau humain. N'est-elle pas au contraire liée à des pratiques toujours plus changeantes?

Mais le facteur le plus important qui explique la faiblesse de cette postérité tient à un changement majeur de conjoncture à partir de la fin des années 1960. C'est durant cette période que l'on assiste à un retournement tant au niveau de l'histoire des idées qu'à celui de l'histoire de l'urbanisme. D'un côté, la doxa techno-scientifique perd son statut hégémonique au sein du monde scientifique. Lié à ce basculement paradigmatique, on constate durant la même période que la profession des planners perd rapidement du terrain notamment suite à la contestation de plus en plus importante des grands projets de rénovations urbaines. De fait, les architectes armés d'un ensemble hétérogène de nouveaux savoir-faire regroupés sous le vocable urban design vont peu à peu reprendre place sur la scène urbaine. C'est notamment ce retournement de conjoncture qui explique que l'urban design ne se constituera jamais comme une discipline mais restera une pseudo-discipline ambiguë ${ }^{24}$. Les approches les plus radicales seront marginalisées alors que les pratiques et modèles Beaux-Arts et paysagistes seront redécouverts.

Où donc chercher la postérité des travaux de ces deux auteurs? La réponse est à la fois étonnante et pourtant évidente: du côté des disciplines dont ils étaient les plus proches. Ainsi The Image of the City est

24. Sur l'état actuel de l'urban design, voir Ali Madanipour, «Ambiguities in Urban Design », Town Planning Review, vol. 68, n³ July 1997, p. 363-383. 


\section{Labyrinthe, $n^{\circ} 20$}

très vite devenu un classique de la psychologie. Une postérité que Kevin Lynch dénonça peu de temps avant sa mort ${ }^{25}$. Les travaux utilisant sa méthode de cartographie de l'image mentale sont presque tous issus de cette science ${ }^{26}$. Quand à Christopher Alexander, c'est à travers ses travaux fortement inspirés de la cybernétique naissante qu'il est aujourd'hui considéré comme un des pères de l'Internet. Un intéressant exemple de traduction de discipline à discipline qu'a permis l'approche interdisciplinaire du cognitivisme.

25. «Reconsidering The Image of the City», dans Tribid Banerjee \& Michael Southworth (dir.), City Sense and City Design. Writings and Projects of Kevin Lynch, Cambridge (Mass.), MIT Press, 1990, p. 247-256.

26. Voir par exemple Kyriaki Tsoukala, L'Image de la ville chez l' enfant, Paris, Anthropos, 2001. 